\title{
Debatte über Griechenland verfehlt Kern von Finanzkrisen
}

Wer in den letzten Tagen die veröffentlichte Meinung in Deutschland zur Situation der Europäischen Währungsunion (EWU) verfolgt hat, muss den Eindruck haben, die Vereinigten Staaten sind ökonomisch am Ende. Denn die USA haben nach Lehman Brothers alle Maßnahmen getroffen, die nicht erst seit dem zweiten Maiwochenende als „Tabubruch“ oder „Sprengsatz" der EWU bezeichnet werden. Die Federal Reserve stockte ihr Portfolio an Staatsschuldtiteln weiter auf und erwarb Papiere mit zweifelhaftem Wert, um die Finanzmärkte zu stabilisieren. Die US-Regierung schnürte ein großes Hilfspaket für Banken mit unsicherer Solvenz. Zur Konjunkturstützung wurden Neuverschuldung und Schuldenstand wesentlich stärker ausgeweitet als in der EWU. Konsequenterweise wurde in den deutschen Medien noch vor wenigen Monaten nicht über die Stabilität des Euro, sondern über die Rolle des US-Dollars als internationale Währung spekuliert. Heute schicken sich die USA an, die Rezession schneller als Europa zu überwinden. Wie lässt sich dieser Widerspruch erklären?

Der deutschen Mehrheitsmeinung zu „Lehman“, „Griechenland“ und dem jüngsten EU-Rettungspaket liegt die Überzeugung zugrunde, dass es sich in allen Fällen um ein reines Überschuldungsproblem handelt. Griechenland hat den Zugang zum Kapitalmarkt verloren, weil es durch hohe Defizite und Staatsschulden insolvent geworden ist. Die Märkte konnten dies nicht erkennen, weil gefälschte Zahlen vorlagen. Griechenland zu helfen, bedeutet daher, den Marktmechanismus auszuhebeln, der bei jeder normalen Unternehmensinsolvenz greift. Zudem droht Inflation, sofern die Zentralbank unterstützend interveniert. Nicht zuletzt aus diesem Grunde wurde im Maastrichter Vertrag die „no bail out“-Klausel aufgenommen. Diese Argumente reflektieren eine theoretische Sichtweise, in der der Kapitalmarkt effizient ist. Finanzkrisen können nicht vorkommen, weil Investoren die Solvenz ihrer Schuldner stets richtig einschätzen. In diesem Theoriegebilde vollständiger und symmetrisch verteilter Informationen kann es keine Ansteckungseffekte geben, können solvente Schuldner nicht illiquide werden, und wirkt eine aktive Geldpolitik inflationär. Auch bei der US-Entscheidung über Lehman dürften diese Überlegungen eine große Rolle gespielt haben.

Die Ereignisse seit dem Sommer 2007 haben gezeigt, dass diese Sichtweise zu kurz greift. Die Finanzkrise brach nicht aus, weil Schuldner insolvent waren, sondern weil es begründete Zweifel an ihrer Solvenz gab. So löste vor fast drei Jahren der Rückgang der US-Häuserpreise Zweifel an der Solvenz der „subprime borrowers" aus. Der Markt für „subprime“-Instrumente wurde illiquide. Niemand wollte diese Titel mehr halten, obwohl weder über die Solvenz noch die Insolvenz der einzelnen Kreditnehmer verlässliche Informationen vorlagen. Die SolvenzZweifel sprangen auf die Banken über, da sie in erheblichem Maße "subprime“Titel erworben hatten. Da Banken Fristentransformation betreiben, wurden auch sie illiquide und mussten von den Zentralbanken refinanziert werden. Im Herbst 2008 kollabierte das Finanzsystem. Statt rationaler Solvenz-Analyse wurden Engagements nach dem Motto aufgelöst: wenn Lehman Insolvenz anmelden muss, welche Finanzinstitution ist dann noch sicher? Zentralbanken und Staaten reagierten auf diesen Ansteckungseffekt und den damit verbundenen Anstieg der Geldnachfrage, indem sie Liquidität und Garantien bereitstellten. Auch insolvente Banken wurden gerettet, weil jeder weitere Konkurs die Zweifel an der Solvenz des Systems insgesamt bestärkt hätte.

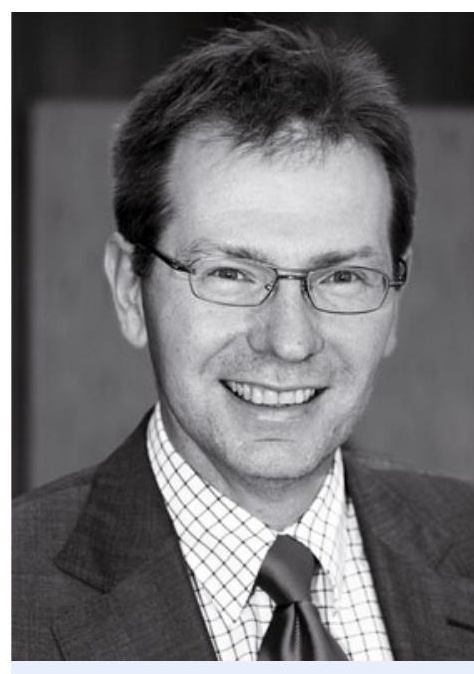

Adalbert Winkler ist Professor für Entwicklungsfinanzierung an der Frankfurt School of Finance \& Management. 
Was hat dies mit „Griechenland“ zu tun? Staaten haben mit Banken gemeinsam, dass sich auch ihre Solvenz nur schwer einschätzen lässt. Typische Kriterien wie Staatsschuldenstand und Neuverschuldung lassen keine eindeutigen Schlüsse zu, sonst müsste Japan größere Finanzierungsprobleme haben als Griechenland. Staaten betreiben auch Fristentransformation. Zwar halten sie keine langfristigen Aktiva, aber im Prinzip gehen Staaten ebenso wie Banken davon aus, dass Altschulden am Kapitalmarkt stets refinanziert werden können. Dass Deutschland, die USA und Japan keine Finanzierungsprobleme haben, liegt daran, dass an ihrer Solvenz nicht gezweifelt wird. Genau dies gilt für Griechenland nicht mehr: die Kapitalmärkte haben das Vertrauen in seine Solvenz verloren, wozu die hohen und gefälschten Defizitzahlen erheblich beigetragen haben. Griechenland hat ein Staatsschuldenproblem, aber das griechische Problem kann nicht allein als Staatsschuldenproblem gesehen werden. Dies zeigt ein Blick auf die Entwicklung spanischer Staatsschuldtitel. Denn Spanien hat vor der Finanzkrise deutlich niedrigere Haushaltsdefizite - in einigen Jahren sogar Überschüsse - erzielt, und stets korrekt berichtet.

Wieso konnte dann „Griechenland“ das europäische Lehman Brothers werden, wie es Barry Eichengreen jüngst ausgedrückt hat? Der Grund liegt darin, dass Griechenland das Symbol für das europäische Wachstumsmodell geworden ist, das bis 2008 - und zwar weit über die EWU-Grenzen hinaus - dem Kontinent eine positive wirtschaftliche Entwicklung ermöglichte. Deutschland und andere Länder im Nordwesten betrieben ein vom Export gestütztes Wirtschaftswachstum, während die Länder im Süden und Osten Wachstum auf der Basis einer von Kapitalzuflüssen forcierten Binnennachfrage bei entsprechend hohen Leistungsbilanzdefiziten erzielten. Europa war eine kleinere Ausgabe dessen, was weltwirtschaftlich als globale Ungleichgewichte mit den USA als zentrales Defizit- und China als zentralem Überschussland diskutiert wurde.

Dieses Modell ist mit dem Versiegen der Kapitalströme nach Lehman im Herbst 2008 zusammengebrochen. Im Gegensatz zu den USA scheinen die europäischen Defizitländer die Rezession aber nicht zu überwinden. Damit wachsen jedoch die Schuldenlasten von öffentlichem und privatem Sektor in den Defizitländern und damit die Zweifel an deren Solvenz. Dies ist der Kern der Finanzkrise, auf die das Hilfspaket für Griechenland keine Antwort gab. Das jüngste Rettungspaket signalisiert, dass das Problem erkannt ist. Es ist deshalb ausdrücklich zu begrüßen. Der Vorwurf, dass damit Grundsätze der EWU gebrochen werden, übersieht, dass das Szenario einer Finanzkrise im Maastrichter Vertrag gar nicht thematisiert wird. Im Mittelpunkt des Vertrages steht ein durch Staatsschulden erzeugtes Inflationsproblem. Dieses Szenario war in den Boomjahren 2006 und 2007 durchaus relevant, als Griechenland und andere EWU-Staaten mit hohen Wachstumsraten eine prozyklische Fiskalpolitik betrieben. Die EZB reagierte und erhöhte die Zinsen. Eine Finanzkrise ist aber durch deflationäre Tendenzen gekennzeichnet, weil sie eine Überschussnachfrage nach Geld impliziert. Auf diese Überschussnachfrage muss die Zentralbank ebenfalls reagieren, wenn sie Preisniveaustabilität erhalten, also Deflation verhindern will.

Dennoch: das Rettungspaket löst das europäische Wachstumsproblem nicht. Es gibt nur eine klare Antwort, wie es nicht gelöst werden soll, nämlich dem US-Beispiel folgend, dass die vormals kreditgetriebene private Nachfrage durch eine kreditgetriebene staatliche Nachfrage in den Defizitländern ersetzt wird. Wie ein neues

Adalbert Winkler

Frankfurt School of Finance \&

Management

a.winkler@frankfurt-school.de Wachstumsmodell aussehen soll, bleibt offen. Vielleicht lohnt sich dabei doch noch einmal der Blick auf die USA. Dort hat die ökonomische Aktivität auch von den expansiven Maßnahmen und der raschen Konjunkturerholung in den globalen Überschussländern profitiert. 